
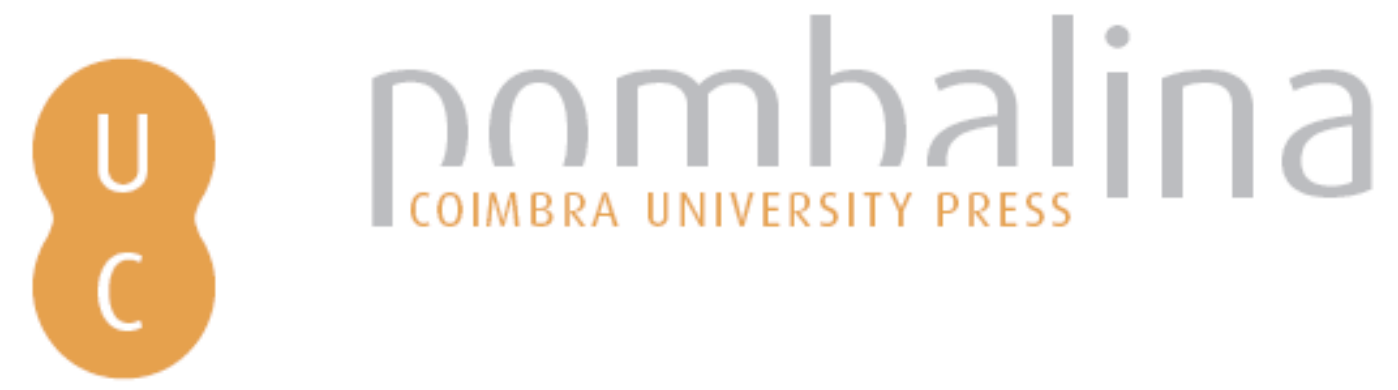

\title{
Construyendo el perfil por competencias del profesional de la información a partir de un dialogo permanente con la sociedad
}
Autor(es):
Pirela Morillo, Johann; Portillo, Lisbeth
Publicado por: Imprensa da Universidade de Coimbra
URL
persistente:
URI:http://hdl.handle.net/10316.2/31966
DOI:
DOI:http://dx.doi.org/10.14195/978-989-26-0319-3_41
Accessed : $\quad$ 26-Apr-2023 13:36:06

A navegação consulta e descarregamento dos títulos inseridos nas Bibliotecas Digitais UC Digitalis, UC Pombalina e UC Impactum, pressupõem a aceitação plena e sem reservas dos Termos e Condições de Uso destas Bibliotecas Digitais, disponíveis em https://digitalis.uc.pt/pt-pt/termos.

Conforme exposto nos referidos Termos e Condições de Uso, o descarregamento de títulos de acesso restrito requer uma licença válida de autorização devendo o utilizador aceder ao(s) documento(s) a partir de um endereço de IP da instituição detentora da supramencionada licença.

Ao utilizador é apenas permitido o descarregamento para uso pessoal, pelo que o emprego do(s) título(s) descarregado(s) para outro fim, designadamente comercial, carece de autorização do respetivo autor ou editor da obra.

Na medida em que todas as obras da UC Digitalis se encontram protegidas pelo Código do Direito de Autor e Direitos Conexos e demais legislação aplicável, toda a cópia, parcial ou total, deste documento, nos casos em que é legalmente admitida, deverá conter ou fazer-se acompanhar por este aviso. 
Maria Manuel Borges

Elias Sanz Casado

Coordenação

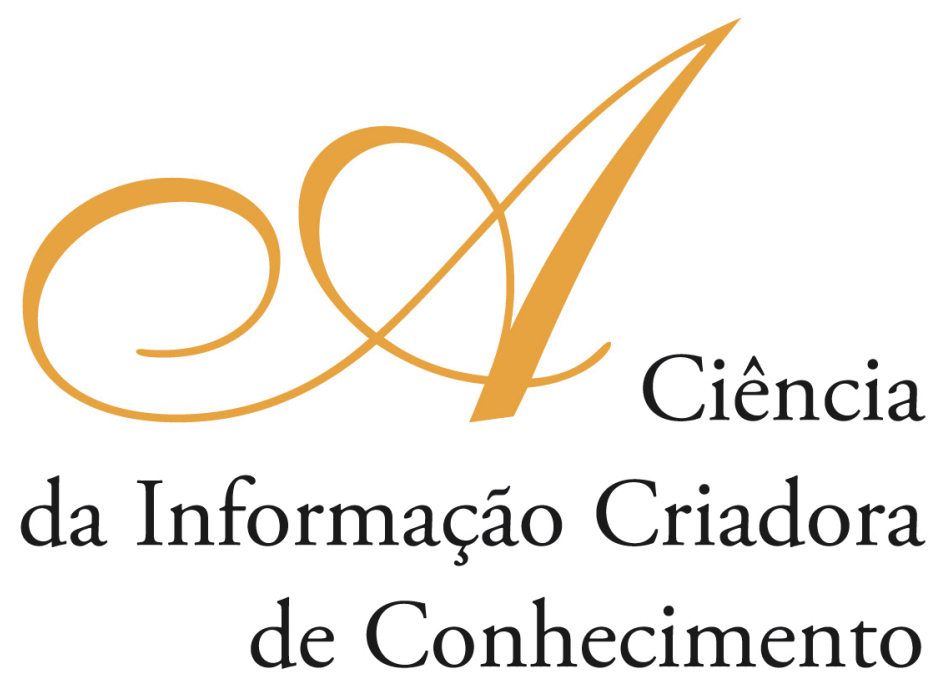

Vol. I

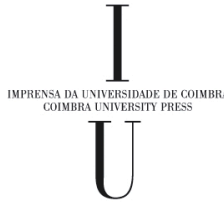

- COIMBRA 2009 
CONSTRUYENDO EL PERFIL POR COMPETENCIAS DEL PROFESIONAL

DE LA INFORMACIÓN A PARTIR DE UN DIALOGO PERMANENTE CON LA SOCIEDAD

\author{
Johann Pirela Morillo \\ Universidad del Zulia (Venezuela) \\ Lisbeth Portillo \\ Universidad del Zulia (Venezuela)
}

\title{
Resumo
}

Se construye y valida el perfil por competencias del profesional de la información de la Escuela de Bibliotecología y Archivología de la Universidad del Zulia, a partir del diseño y ejecución de una metodología -Tecnología cooperativa-, orientada fundamentalmente a estimular y propiciar a través de un diálogo permanente con la sociedad, la participación activa no solo de los actores que forman parte de la dinámica curricular (profesores, estudiantes, egresados y los diseńadores y planificadores del currículo), sino también de representantes estratégicos de los distintos sectores sociales. Dicha metodología se operacionaliza mediante el desarrollo de tres fases a saber: 1) La Arquitectura conceptual, fundamentada por las actuales corrientes y tendencias que orientan los cambios de la educación superior para el siglo XXI, principalmente en lo que respecta a la concepción del currículo como concreción de una teoría pedagógica planteada por Inciarte (2005), y del Currículo Cooperativo Incardinado en la Sociedad propuesto por Rincones (2007). 2) Consulta de los actores medulares, con esta fase se logró obtener la visión y aportes de los distintos actores de la sociedad, con relación a la formación de ls profesionales de la información, y 3) Revisión de estudios de mercados que permitieron detectar las necesidades reales, potenciales y emergentes del mercado laboral en varios países de América Latina. Como resultado de la aplicación de esta metodología se concretó el el perfil del profesional de la información atendiendo a cuatro campos de competencias: el de la gerencia de la información y del conocimiento, el de la mediación de la información, el de la organización y representación de la información y el conocimiento y el de la promoción socio-cultural. Se concluye que sólo es posible garantizar la pertinencia de los perfiles profesionales basados en competencias si éstos se construyen sobre la base de la participación interactiva de la sociedad.

\begin{abstract}
It is constructed and validated the profile for competitions of the professional of the information of the School of Library and Archives of the University of Zulia, starting from the design and execution of a methodology (cooperative technology) orientated, fundamentally, to stimulate and to propitiate through a permanent dialog with the society, the active participation of the actors that form part of the curricular dynamic (professors, students, graduated, designer and planners of the curriculum), as well as the strategic agents of the different social sectors. Mentioned methodology, it is executed through the development of three faces to know: 1) the Conceptual Architecture, based on the current flows and tendencies that orientate the changes
\end{abstract}


of the superior education for the XXI century, with respect to the conception of the curriculum as materialization of a pedagogical theory established by Inciarte (2005), and of the Incardinated Cooperative Curriculum in the society proposed by Rincones (2007). 2) The Consult of the medullar actors, with this face, it was obtained the vision and contributions of the different actors of the society, with relation to the formation of the professionals of the Information, and 3) Revision of studies of markets that allows to detect the emergent, potential and real necessities of the labor market in countries of Latin American. As result of the application of this methodology, it was materialized the profile of the professional of the Information, attending four fields of competitions: management of the information and of the knowledge, mediation of the information, organization and representation of the information, knowledge and of the social-cultural promotion. It is concluded, that is only possible to guarantee the pertinence of the professional profiles based on competitions, if these are constructed on the base of the interactive participation of the society.

\section{Introducción}

Para la construcción y validación del perfil por competencias del profesional de la información se consideró como premisa que ante la complejidad y el dinamismo de la sociedad actual se requiere repensar el currículo de las Escuelas de Bibliotecología, Archivología y Ciencia de la Información de América Latina, ya que éste debe articularse en torno a la posibilidad de formar profesionales de la información que contribuyan, de manera decisiva, con la construcción de una sociedad incluyente del conocimiento.

Bajo estos principios, se identificaron dos ejes para la fundamentación teórica que sirviera de marco orientador en el diseño del perfil por competencias del profesional de la información. Por un lado, las características del nuevo contexto de la sociedad y, por el otro, las tendencias educativas que de ese contexto emergen y cómo han sido interpretadas, interiorizadas y expresadas en los diseños curriculares de las Escuelas de Bibliotecología, Archivología y Ciencia de la Información.

Fue necesario considerar además, las necesidades reales, potenciales y emergentes del mercado laboral, de los distintos grupos comunitarios de la sociedad, con el objeto de que el perfil de los profesionales diera respuesta a los requerimientos del entorno social y permitieran consolidar procesos de evaluación y acreditación de los planes y programas, como una forma de garantizar su calidad.

Sobre la base de estos planteamientos, se consolidó una tecnología cooperativa (Pirela, 2008), que integra el análisis conceptual con técnicas de investigación cualitativas e interactivas tales como la técnica del taller, el encuentro interactivo y el conversatorio que permitieron develar las visiones de los diferentes actores sociales. Esta metodología plantea las tres fases fundamentales contempladas para el diseño curricular de la Escuela de Bibliotecología y Archivología de la Universidad del Zulia. La primera fase, la Arquitectura conceptual recoge el marco teórico que fundamenta el currículo, considerando las distintas tendencias educativas y de servicios de información.

Este diseño se elaboró a partir de la caracterización del enfoque curricular que hemos denominado cooperativo y redificado, el cual se asume como un proceso complejo, dinámico, abierto, participativo, intersubjetivo e interdiscursivo, que tiene sentido en un momento histórico y socio-pedagógico que debe generar un producto innovador 
que trascienda lo técnico y de respuestas a las cambiantes y crecientes necesidades del mundo del trabajo y las particularidades de la profesión.

Tecnología cooperativa. Una metodología para el diseño del perfil por competencias del profesional de la información

La Tecnología cooperativa constituye una metodología definida por (Pirela, 2008) como un proceso pensado, generado y validado a partir de la reflexión y la acción colectiva, orientada hacia la creación de comunidades articuladas sinérgicamente por valores comunes, las cuales proyectan y construyen visiones prospectivas, concretándolas desde el presente.

Dicho concepto se apoya en la perspectiva de Páez (1992), relacionada con la tecnología social y la tecnología intelectual, en la idea de relación entre los "sociosistemas" y las tecnologías, de González, López y Luján (2004) y en la noción de Picón y otros (2005), sobre el planteamiento de una Tecnología Social de Mediación (TSM).

En este sentido, la tecnología cooperativa (grafico No. 1) para el diseño del perfil por competencias del profesional de la información, se define como un conjunto de concepciones que fundamentan la acción reflexiva, orientada hacia la evaluación y diseño curricular en el área. La tecnología se apoya en el uso crítico de recursos de tecnología instrumental de información y comunicación para sistematizar los significados, hacer seguimientos y monitoreos permanentes, integrar y consolidar resultados.

Esta metodología, en su dimensión operativa se concreta en tres fases de realización que incluyen procedimientos de análisis teórico y crítico, de estudio de tendencias, el cotejo de estudios de mercado y la participación abierta y colectiva en torno al futuro de la formación del profesional de la información, desde la visión de los egresados, estudiantes, representantes del sector empleador y grupos comunitarios organizados. Todo ello sin dejar de lado la fundamentación teórica, epistemológica y axiológica que guió el proceso de concepción y ejecución de cada una de las etapas, procesos y productos de dicha tecnología.

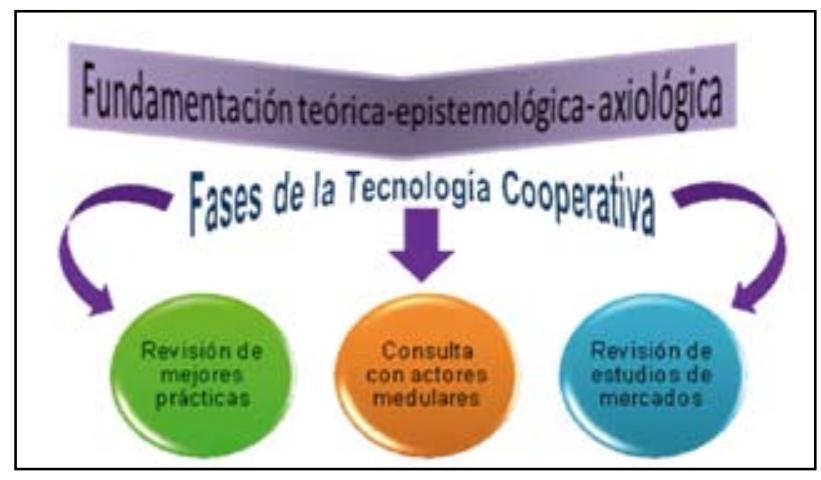

Figura 1 - fases de la tecnología cooperativa 
Fases de un modelo curricular cooperativo y redificado, construido sobre la base del diálogo permanente con la sociedad .

Fase 1. Construcción de la arquitectura conceptual.

La arquitectura conceptual del modelo se estructuró mediante la integración de diversos enfoques: a) las características de la sociedad actual, b) las tendencias de la educación superior del Siglo XXI, y c) las tendencias de los servicios de información en el mundo.

a) Caracterización de la sociedad actual: la sociedad del conocimiento, de la comunicación y del aprendizaje.

La idea de avanzar hacia una sociedad del conocimiento, la comunicación y el aprendizaje sin exclusiones se apoya en planteamientos que sostienen que el desarrollo individual y social no es posible solamente con garantizar el acceso a la información y sus tecnologías, sino, también, en que es importante educar al sujeto en el uso "crítico" y "estratégico" de los contenidos para construir conocimiento que pueda utilizarse en la toma de decisiones y en la resolución de problemas de la vida académica, profesional y cotidiana. Se trata de desplazarse del documento a la información y de ésta al conocimiento, con el propósito de generar participación creativa de los ciudadanos mediante la adquisición de competencias para aprender a lo largo de toda la vida.

En palabras de Morales (2003), el acercamiento al conocimiento es posible por la vía de la información y la educación; pero esto requiere de procesos mentales complejos, así como también de procesos sociales y culturales, de modo que el salto cualitativo que se debe dar entre contar documentos organizados y con información sistematizada para construir conocimiento útil (mediado por las nuevas formas de comunicación digital) debe basarse en el aprendizaje de las habilidades y destrezas de agregación de valor a la información y el desarrollo de competencias, con el fin de interactuar de una manera efectiva en contextos comunicacionales mediados por tecnologías telemáticas.

La sociedad del conocimiento no existe en la medida en que se exprese en el plano declarativo y argumentativo por grupos de intelectuales, especialistas en información o asociaciones internacionales, tal y como lo plantea Morales (2003), sino que en este contexto de la transición de la sociedad de la información a la del conocimiento, la comunicación y el aprendizaje, se requiere fundamentalmente el esfuerzo mancomunado de académicos, profesionales de la información y, sobre todo, del interés de organismos gubernamentales en materia de formulación de políticas, programas y estrategias que incluyan, por supuesto, las propuestas de formación académica de los profesionales de la información como agentes fundamentales para la construcción colectiva de la sociedad incluyente del conocimiento (Cumbre Mundial sobre la Sociedad de la Información. Primera Fase, 2003).

b) Tendencias de la Educación Superior en el siglo XXI

Desde el conocido informe Delors . UNESCO (1996), se ha planteado la necesidad de repensar la educación en todos los niveles y modalidades, con el propósito de ajustar los proyectos de formación de las instituciones educativas a las cambiantes y complejas 
necesidades de un mundo cada vez más signado por la turbulencia y la incertidumbre. Estas ideas han tomado nuevos matices y se les ha dado un mayor énfasis considerando los rasgos y características de la posible transición hacia la sociedad del conocimiento, la comunicación y el aprendizaje.

En la Declaración Mundial sobre la Educación Superior UNESCO (1998), se insiste en responder a los nuevos desafíos que se le imponen a los procesos de formación de los cuadros profesionales que se requieren para impulsar el desarrollo.

En la Cumbre Mundial sobre la Sociedad de la Información (CMSI 2005), uno de los planteamientos fue precisamente transitar hacia la sociedad del conocimiento centrada en la persona, mediante la vinculación de los sistemas electrónicos de información y las redes globales con sus diferentes contextos de acción. Por su parte, también la (UNESCO, 2005) propuso el papel estratégico que tiene la educación superior para contribuir con la construcción de la sociedad del conocimiento, mediante la formación de redes de enseñanza que busquen establecer puentes que permitan una comunicación fluida entre las instituciones educativas y los sectores sociales.

En este sentido, se ha venido insistiendo sobre la necesidad de introducir cambios en la Educación Superior. Estos cambios y tendencias apuntan hacia los siguientes aspectos centrales:

1. Concepción del currículo como concreción de una teoría pedagógica y como un plan en permanente construcción. Inciarte (2005), Flórez (2001), Marcano (2000), y bajo la concepción del currículo cooperativo redificado de Rincones, (2006) (2007)

2. Estructura de los perfiles profesionales con base en competencias. que integren no sólo los conocimientos teóricos y procedimentales, sino también los axiológicos y actitudinales Gonzci y Athanason, (1996), Díaz-Barriga y Rigo (2002), Cánquiz, (2005), Muñoz (1998), Muñoz y Rubiano (1998), Cornella (1999), Gómez-Fernández (2002), Cano (2002), Vieira da Cunha y otros (2004), Barber (2005), Pirela y Peña (2005)

3. Garantizar la pertinencia y la calidad en los planes y programas de estudio. Declaración Mundial sobre la Educación Superior. UNESCO (1998) Tunnermann (2002), Comisión Nacional de Evaluación y Acreditación Universitaria CONEAU (1995), Malo (2005), Comités Interinstitucionales de Evaluación de la Educación Superior (CIEES) y el Consejo para la Acreditación de la Educación Superior (COPAES)

4. Asumir nuevos modelos pedagógicos centrados en el aprender a aprender y en el desarrollo de procesos del pensamiento. documento "Compromiso de Túnez", generado por la Cumbre Mundial sobre la Sociedad de la Información. CMSI (2006) y las ideas de UNESCO (2005),

5. Introducir las tecnologías de información y comunicación como eje de conocimiento, como eje transversal y como nueva forma de conducir los procesos de aprendizaje.

6. Concebir la investigación como eje de conocimiento y como eje transversal, bajo la modalidad transdisciplinaria a modo de vía para abordar la complejidad desde el currículo.

Es importante resaltar que dentro de las 6 tendencias declaradas anteriormente, el modelo que se propone esta fundamentado esencialmente en la primera de ellas, es decir en la concepción del currículo como concreción de una teoría pedagógica y como un plan en permanente construcción. Inciarte (2005), Flórez (2001), Marcano 
(2000), y bajo los principios que sustentan el enfoque curricular cooperativo e incardinado de Rincones, (2006) (2007), cuyas características se especifican en el siguiente apartado.

c) Tendencias de los servicios de información en el mundo

Conscientes de las dimensiones de la nueva sociedad que se busca construir, se plantea la necesidad de contar también con servicios de información articulados en torno a las demandas informativas, formativas y recreativas de las personas, grupos y organizaciones. En este sentido, se consideran las tendencias que han proyectado organismos como IFLA y UNESCO, en relación con la naturaleza, alcance y propósitos de los nuevos servicios de información.

Se espera que tales servicios garanticen a los ciudadanos el acceso equitativo a la información que se produce, como una forma de reducir las posibles brechas sociales, cognitivas y digitales. Para ello, se requiere que los servicios de información se entiendan como organizaciones de conocimiento que deben tener un rol protagónico en la formación de las personas, grupos y organizaciones para la inserción activa en la sociedad. Las concepciones, funciones y procesos que estas organizaciones deben desarrollar estarán centradas en los usuarios quienes necesitan saber utilizar la información, el conocimiento y las tecnologías digitales para impulsar su desarrollo personal-social y profesional.

\section{Fase 2: Consulta de los actores medulares. Sondeo de opiniones}

Por medio de esta fase logró estimular y propiciar la participación de los actores medulares para la definición del perfil por competencias del profesional de la información. Para ello se consideró la generación de espacios de diálogo intersubjetivo, mediante la instrumentación de técnicas cualitativas de investigación como el taller interactivo y el conversatorio. Los espacios de diálogos se concretaron a través de: a) la organización de la IV Jornada de Evaluación Curricular y Organizacional de la Escuela, donde participaron los profesores, diseñadores y planificadores del currículo; b) el II Encuentro de egresados de la Escuela, donde además de la asistencia de los egresados, se invitaron a estudiantes, miembros del sector empleador y representantes de algunos sectores de la comunidad, es decir que dentro del marco de este ultimo evento se logró obtener, a su vez importantes opiniones de estos sectores.

Con la implantación del Servicio Comunitario en las Universidades venezolanas y la inserción de alumnos y profesores de la Escuela de Bibliotecología y Archivología de la Universidad del Zulia en proyectos de impacto social, se han venido detectando vacíos en la formación de los profesionales de la información, sobre todo en aspectos atinentes a la dimensión social y comunitaria del manejo de la información y de la lectura. Por ello, el servicio comunitario ha permitido establecer diálogos permanentes con la sociedad y a partir de estos diálogos se han tomado decisiones relacionadas con la inclusión en el perfil profesional de competencias dirigidas a consolidar el rol de promotor socio-cultural del profesional de la información.

Cabe destacar que el Servicio Comunitario se rige por la Ley de Servicio Comunitario del Estudiante de Educación Superior, aprobada en el año 2005 para incorporar en 
todas las carreras de las Universidades nacionales la dimensión comunitaria, la cual se concreta mediante el cumplimiento por parte de los estudiantes de 120 horas que deben dedicar al desarrollo de un proyecto comunitario, previamente aprobado por la Facultad, Escuela y/o Departamento de la Universidad.

En el caso de la Escuela de Bibliotecología y Archivología, algunos de los proyectos, por medio de los cuales se ha generado el diálogo con grupos comunitarios, son: Lectura recreativa en la comunidad durante el período vacacional y Promoción y animación de lectura en Escuelas Públicas Nacionales; éste último proyecto se desarrolló por medio de un convenio firmado con la Alcaldía de Maracaibo.

Con el desarrollo de estos proyectos se han podido establecer relaciones permanentes con actores sociales fundamentales, quienes han expresado la necesidad de formarse como lectores críticos, mediante la consolidación de habilidades para el aprendizaje de de la información y la lectura como herramientas para la construcción del conocimiento y como instrumento que permite potenciar la participación y cohesión social.

De manera que este diálogo con la sociedad, posible gracias a los proyectos de servicio comunitario del estudiante de la Escuela de Bibliotecología y Archivología, ha venido justificando cada vez más,el reforzamiento del rol de promotor socio-cultural del profesional de la información.

Fase 3. Revisión de estudios de mercados de trabajo en Bibliotecología, Archivología y Ciencias de la Información. Una visión internacional

En el área de Bibliotecología y Ciencia de la Información se han realizado estudios de egresados y de mercado de trabajo que han asumido distintas metodologías y técnicas de investigación, tal como lo encontraron Escalona y Fernández (2007) y Pirela (2008). Los estudios realizados exploran variables y aspectos disímiles, mientras unos se abocan a esclarecer las fortalezas y debilidades de la formación profesional, al tiempo que procuran acercarse a los sectores empleadores para definir la demanda real, otros, en cambio exploran rasgos propios de los perfiles socio-laborales y ocupacionales de los profesionales, haciendo énfasis en la naturaleza, evolución y proyección de la inserción en el mercado de trabajo. Otros estudios de egresados y de mercado de trabajo se centran más en identificar los sectores con mayor penetración de profesionales de la información y las funciones y actividades más realizadas.

Los estudios a los cuales se hace referencia fueron realizados en Argentina por Pineda (2002), en Colombia por Delgado (2005) y la Asociación Colombiana de Bibliotecólogos y Documentalistas ASCOLBI (2006), en España por Moreiro (2006), Rey y Atenas (2008), García, Ortoll y Corbasi (2008) y en Venezuela, de modo preliminar, por Pirela y Portillo(2008).

La integración de las tres fases anteriores, permitió fundamentar teórica, epistemológica y axiológica el modelo curricular cooperativo y redificado que se concreta en un nuevo perfil del profesional de la información con base en competencias integradas y organizadas por ejes curriculares-cognoscitivos. 
Perfil por competencias del profesional de la información de la Escuela de Bibliotecología y Archivología de la Universidad del Zulia.

La composición del perfil profesional por competencias se muestra en una versión gráfica (Ver Gráfico No.2.), en las cuales se organizan las competencias genéricas propuestas por la Comisión de Currículo de la Universidad del Zulia para todas las carreras, las competencias básicas y las competencias específicas, éstas últimas son resultado de la integración de los aportes derivados de las fuentes y actores consultados.

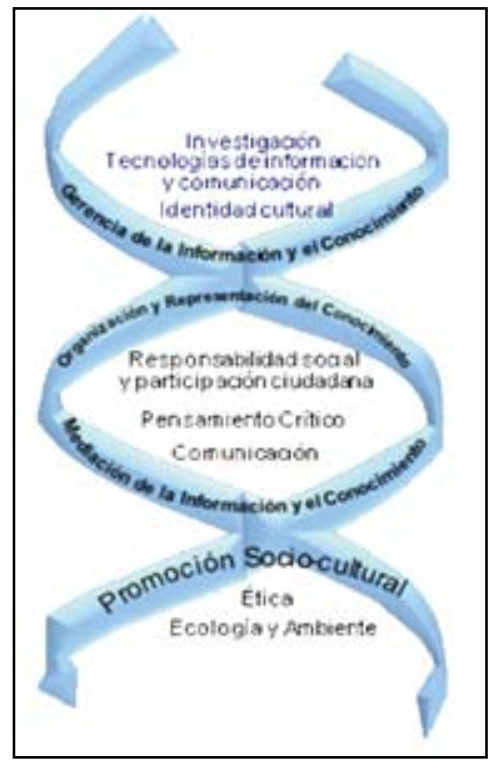

Figura 2 - Composición del perfil por competencias del profesional de la Información

El perfil por competencias del profesional de la información definido en la Escuela de Bibliotecología y Archivología de la Universidad del Zulia asume como referente simbólico la cadena de AND para representar la filosofía del enfoque de competencias, que cual plantea la confluencia de diferentes niveles en el dominio de saberes y desempeños en el marco de la formación integral. Las competencias se dividen en genéricas, básicas y específicas y apuntan hacia un profesional de la información que contribuya con la reducción de la brecha digital y social, en el contexto de la gestión de la información y el conocimiento y la alfabetización informacional, como estrategias transversales.

El perfil se organiza en función de desarrollar cuatro campos de competencias: gerencia de la información y del conocimiento, organización y representación de la información y el conocimiento, mediación de la información y promoción sociocultural. Estos campos se apoyan en el dominio de competencias genéricas, relacionadas con la ética, la ecología y el ambiente, la comunicación, el pensamiento crítico, la responsabilidad social y participación ciudadana, la identidad cultural, el manejo de las tecnologías de la información y la comunicación y la investigación. 


\section{Referências bibliográficas}

AMERICAN LIBRARY ASSOCIATION ALA. El Código de Ética de la Asociación de Bibliotecas de los Estados Unidos. 1995. (Versión en español). En http://www.ala.org/ala/ oif/statementspols/codeofethics/ coespanishversion/spanishversion.htm [Consultado en septiembre de 2006].

Asociación Colombiana de Bibliotecólogos y Documentalistas. (ASCOLBI). (2006). Análisis de la situación laboral del Bibliotecólogo en Colombia. Disponible en línea: www.ascolbi. org/ documentos/Situación_Laboral2006.pdf. (Consulta: 09- 01-08).

Barber, E. (2005) La enseñanza de la Bibliotecología y Ciencia de la Información: situación en las universidades argentinas. En: Martínez Arellano, F.F. y Calva González, J.J. (Comps). Seminario INFOBILA como apoyo a la investigación y educación en Bibliotecología en América Latina y el Caribe. págs 3-16. México. Universidad Nacional Autónoma de México. UNAM. Centro Universitario de Investigaciones Bibliotecológicas. CUIB.

Canquiz-Rincón, L. (2005) Perfiles profesionales basados en competencias. ponencia presentada en el Foro: Hacia una nueva visión del currículo en LUZ. Universidad del Zulia. ViceRectorado Académico. Maracaibo-Venezuela. 27 y 28 de octubre de 2005.

Cano, V. (2002). De bibliotecario a gestor de información. ¿Cambio de nombre o nuevas competencias?. En: http://jimmy,qmced.ac.uk/usr/imres/ fulltxt/txt_VC3.htm. (Consulta: 08-11-07).

Comisión Nacional de Currículo (CNC), (2001). Lineamientos para abordar la transformación en la Educación Superior. Escenarios curriculares. En: Agenda académica. Vol. 8, No. 1. Disponible en línea: http://150.185.136.100/pdf/agenda/v8n1/articulo5.pdf. (Consulta: 02-09-07).

Cornella, A. (1999). Extra! Net. Mensaje 440(25-06-99). En: http://www.extra-net.net/articulos/ en990625.htm. (Consulta: 08-11-2007).

Cumbre Mundial sobre la Sociedad de la Información. (CMSI) (2006). Compromiso de Túnez. Disponible en línea: http://www.itu.int/wsis /docs2/tunis/off/7-es.html. (consulta: 03-11-08)

Delgado, A (2005). Aportes para el estudio del ejercicio profesional del bibliotecólogo en Colombia. http://eprints.rclis.org/archive/ 00005023/01 acreditabibliotecolombia.pdf. (Consulta: 12-12-07)

Díaz-Barriga, F y Rigo, M.A. (2002) "Formación docente y educación basada en competencias" En: Valle, María de los Ángeles. (coord.) Formación en competencias y certificación profesional. págs 17-44. México. Universidad Nacional Autónoma de México. Centro de Estudios sobre la Universidad.

Díaz-Barriga, F. y Hernández, G. (2002) Estrategias docentes para un aprendizaje significativo. Una interpretación constructivista. Mc. Graw Hill. México-DF. 465 p.

Escalona, L. y Fernández, E. (2007) Los estudios de egresados del área bibliotecológica: de la teoría a la práctica. Revista Interamericana de Bibliotecología. Jul-Dic. 2007, vol 30, No.2, p. 113-135.

Flórez O. R. (2002). "Currículo y Pedagogía: Nuevas tendencias". Conferencia presentada en la VReunión Nacional de Currículo: Escenarios para la Universidad del Siglo XXI (publicada en memorias), febrero, 19-22. Caracas-Venezuela. 2002.

García, M. Ortosll, E. y Corbasi, J. (2008) Tendencias en el mercado laboral: nuevos perfiles profesionales en Información y Documentación. Ponencia presentada en el III Encuentro Ibérico de Docentes e Investigadores en Información y Documentación. Formación, 
investigación y mercado laboral en información y documentación en España y Portugal. (publicada en Memorias). Universidad de Salamanca-España. p. 479-486..

Gómez-Fernández, J. (2002). El profesional de la documentación. Guiones de la asignatura Midus. En: http://www.arrakis.es/amjg/per9.htm. (Consulta: 09 de noviembre de 2008).

González G. Marta, López C. José A. y Luján L, José (2004). Ciencia, Tecnología y Sociedad: una introducción al estudio social de la ciencia y la tecnología. Reproducido y disponible en línea por: Ciencia, tecnología, sustentabilidad. El Escorial-España. Erro! A referência de hiperlink não é válida..

Gonzci, A. y Athanasou, J. (1996) "Instrumentación de la educación basada en competencias. Perspectiva de la teoría y la práctica en Australia” En: Antonio Argüelles (comp.) Competencia laboral y educación basada en normas de competencia. México, SEP/CNCCL/CONALEP. pp 265-268.

Inciarte, A. (2005) Retos y principios del currículo de la educación superior. Ponencia presentada en el Foro: Hacia una nueva visión del currículo en LUZ. Universidad del Zulia. Vice-Rectorado Académico. Maracaibo- Venezuela. 27 y 28 de octubre de 2005.

Malo, S (2005). Papel de la evaluación y acreditación en la relación entre la universidad y el Estado. Una visión desde el Centro Nacional de Evaluación para la Educación Superior, México. En: Seminario Regional: las nuevas tendencias de la evaluación y de la acreditación en América Latina. En: http://www.coneau.gov.ar/seminario/6\%20 Y\%207/SMalo.pdf. Consulta: 26-09-07

Marcano, N (2000). Coherencia y pertinencia de los diseños curriculares para la formación docente. Un estudio comparativo. Universidad del Zulia: Maracaibo-Venezuela.

Morales, E. (2003). "Comentarios a partir del documento de introducción a la Mesa: Las tecnologías de información y comunicación y la construcción del conocimiento”. En: Almada de Ascensio y otros (Eds). Contribución al desarrollo de la Sociedad del Conocimiento. págs.258265. México. Universidad Nacional Autónoma de México. UNAM. Centro Universitario de Investigaciones Bibliotecológicas. CUIB

Moreiro, J. A. (2006). Influjo de la convergencia europea y de la Inserción laboral en los nuevos planes de estudio de Información y Documentación en España. Conferencia dictada en el Encuentro de Educación e Investigación Bibliotecológica. Lima-Perú. Universidad Mayor de San Marcos, del 6 al 8 de noviembre de 2006.

Muńoz, C. V. (1998). El papel del gestor de la información en las organizaciones a las puertas del Siglo XXI. En: http://www.florida-uni.es/'fesabid98/Comunicaciones/m_munyoz.htm. (Consulta: 08 de noviembre de 2002).

Muñoz, M y Rubiano, P. (1998). El bibliotecario digital: el perfil del nuevo profesional de la información. En: http://www.florida-uni.es/` fesabid98/Comunicaciones/m mundos.htm. (Consulta: 08-11-02).

Páez U, Iraset (1992) Gestión de la inteligencia, aprendizaje tecnológico y modernización del trabajo informacional. Retos y oportunidades. Instituto de Estudios del Conocimiento. Universidad Simón Bolívar. Caracas-Venezuela.

Picón M, G y otros (2005) Cuando la Universidad va a la Escuela. Universidad Pedagógica Experimental Libertador. UPEL. Vicerrectorado de Investigación y Postgrado. CaracasVenezuela.

Pineda, J. M. (2002). Realidad laboral de los bibliotecarios y bibliotecólogos: Egresados de la Escuela de Bibliotecología dependiente de la Facultad de Filosofía de la Universidad Nacional de Córdoba-Argentina. En: Biblios. Revista Electrónica de Ciencias de la Información. 
Disponible en línea: http://eprints.rclis.org/archive/ 00002351/01/B12-01.pdf. Consultado: 08-10-07.

Pirela, J y otros (2008). Centro de Investigación y Desarrollo en Tecnologías del Conocimiento. "Dr. Iraset Páez Urdaneta" CIDTEC. Proyecto de creación. Escuela de Bibliotecología y Archivología. Facultad de Humanidades y Educación. Universidad del Zulia. MaracaiboVenezuela.

Pirela, J. (2008). Tecnología cooperativa para el diseño del perfil por competencias del profesional de la información. Trabajo de Ascenso para optar a la categoría de Profesor Titular de la Escuela de Bibliotecología y Archivología de la Universidad del Zulia.

Pirela, J., (2007). "Las tendencias educativas del Siglo XXI y el currículo de las Escuelas de Bibliotecología, Archivología y Ciencia de la Información de México y Venezuela”. Revista Investigación Bibliotecológica: Archivonomía, biblioteconomía e información, Vol 21, No. 43, julio-diciembre, 2007. págs. 73-105. México.

Pirela, J. y Peña, T. (2005). "Nuevos desafíos para la formación del profesional de la información: un enfoque de competencias". En: Revista Investigación Bibliotecológica: archivonomía, biblioteconomía e información. No. 38, Vol. 19, enero-junio de 2005. págs: 118-139. Centro Universitario de Investigaciones Bibliotecológicas. Universidad Nacional Autónoma de México.

Rey, C. y Atenas, J. (2008) ¿Qué está buscando el mercado laboral? Competencias profesionales y currículum. Ponencia presentada en el III Encuentro Ibérico de Docentes e Investigadores en Información y Documentación. Formación, investigación y mercado laboral en información y documentación en Espańa y Portugal. (publicada en Memorias). Universidad de SalamancaEspaña. p. 479-486.

Rincones, L. (2006) El currículo cooperativo incardinado: trama teórica de sustentación. Ponencia presentada en el 1er. Congreso Nacional de Investigación y Postgrados en Humanidades y Educación y Jornadas Internas de Investigación de la Facultad de Humanidades y Educación de la Universidad del Zulia. (resumen publicado en memorias). Universidad del Zulia. Del 16 al 20 de octubre de 2006. Maracaibo-Venezuela.

Rincones, L. (2007). El currículo cooperativo incardinado. Trama teórica de sustentación. Tesis doctoral (no publicada). Programa de Doctorado en Ciencias Humanas. Universidad del Zulia. Maracaibo-Venezuela.

Tunnermann, C. (2002). Tendencias contemporáneas en la transformación de la educación superior. En: V Reunión Nacional de Currículo: escenarios para la universidad del Siglo XXI. Del 19 al 22 de febrero de 2002. Universidad Central de Venezuela. Caracas-Venezuela.

UNESCO (1996). La educación encierra un tesoro. Informe a la UNESCO de la Comisión Internacional sobre la Educación para el Siglo XXI. Presidida por Jackes Delors. Madrid: Santillana.

(1998). Declaración mundial sobre la educación superior en el Siglo XXI: visión y acción. http://www.unesco.org/education/educprog/wche/declaration_spa.html. (Consultado: 01-09-07).

UNESCO. (2005) Hacia las sociedades del conocimiento. Informe Mundial de la UNESCO. (Disponible en línea) http://unesdoc.unesco.org/ images/0014/001419/141908s.pdf. consulta: $12-02-07$

Vieira da Cunha, M. y otros (2004). "O bibliotecario formado pela Universidade Federal de Santa Catarina”. En: Perspectivas em Ciencia da Informacao . Vol 9, No. 2, julio-diciembre. Págs. 182-195. Brasil-Belo Horizonte. 